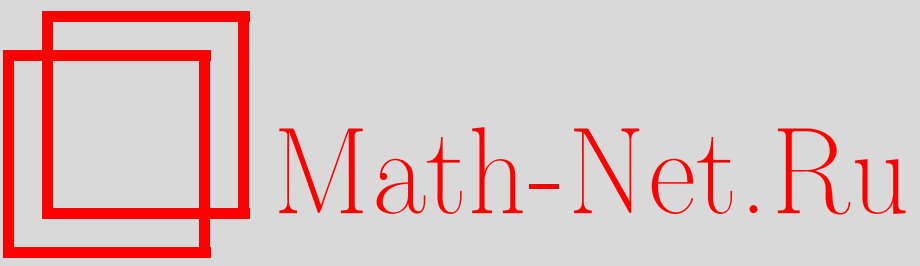

М. Ю. Ковалевский, А. А. Рожков, К теории раствора квантовых фермижидкостей с векторным параметром порядка, ТМФ, 2001, том 127, номер 2, 317 335

DOI: https://doi.org/10.4213/tmf460

Использование Общероссийского математического портала Math-Net.Ru подразумевает, что вы прочитали и согласны с пользовательским соглашением http://www . mathnet.ru/rus/agreement

Параметры загрузки:

IP: 35.173 .137 .237

26 апреля 2023 г., 16:51:49 
ТЕОРЕТИЧЕСКАЯ

И МАТЕМАТИЧЕСКАЯ

ФИЗИКА

Том 127, № 2

май, 2001

(C) 2001 г.

М. Ю. Ковалевский* ${ }^{*}$ А. А. Рожков ${ }^{\dagger}$

\section{К ТЕОРИИ РАСТВОРА КВАНТОВЫХ ФЕРМИ-ЖИДКОСТЕЙ С ВЕКТОРНЫМ ПАРАМЕТРОМ ПОРЯДКА}

Построена термодинамика и дан вывод уравнений гидродинамики раствора сверхтекучих ферми-жидкостей с векторным параметром порядка. Термодинамические величины, связанные со спонтанным нарушением фазовой симметрии и спиновых вращений, представлены в терминах оператора параметра порядка. Предсказан новый эффект увлечения, связанный со взаимным влиянием магнитных и орбитальных степеней свободы. Получены спектры коллективных возбуждений, и обсуждены отличия рассматриваемого состояния от состояний со скалярным и тензорным параметрами порядка.

\section{1. ВВЕДЕНИЕ}

Современные тенденции развития теории квантовых жидкостей связаны с изучением состояний, характеризуюшихся различными параметрами порядка (ПП). Такие конденсированные среды, как сверхтекучая фаза Не-3, многокомпонентная электронная жидкость в 3d-металлах, сверхтекучая ядерная материя, описываются в терминах векторного или тензорного ПП. Наиболее изученные примеры квантовых бозе- и фермижидкостей в сверхтекучем состоянии характеризуются скалярным ПП [1], [2]. В однокомпонентной сверхтекучей ферми-жидкости с триплетным спариванием имеет место спонтанное нарушение симметрии относительно фазовых преобразований и групп вращений в конфигурационном и спиновом пространствах. Эти состояния описываются тензорным ПП [3]-[6]. Построению термодинамики и выводу уравнений гидродинамики таких систем посвяшены работы [3], [4], [7], [8], где, как правило, используются различного рода модельные гамильтонианы.

Явление сверх текучести в случае конденсированных сред с несколькими законами сохранения числа частиц для скалярного ПП изучалось в работах [9]-[11]. Для раствора ферми-жидкостей, помимо скалярных и тензорных ПП, характерных для однокомпонентной жидкости, можно ввести ПП векторного типа в конфигурационном или спиновом пространстве. В этом случае возникают задачи описания равновесия и гидро-

\footnotetext{
* Харьковский физико-технический институт, Харьков, Украина

${ }^{\dagger}$ Научный физико-технологический центр НАН и МО Украины, Харьков, Украина
} 
динамического этапа с векторным ПП, сравнения с другими возможными физическими состояниями со скалярным и тензорным ПП.

Эффективным инструментом построения теории квантовых жидкостей является ферми-жидкостный подход [12], применявшийся для описания сверхтекучих жидкостей с синглетным спариванием [13], триплетным спариванием [14], [15], магнитных систем со спонтанным нарушением симметрии [16]. Ферми-жидкостный подход для многокомпонентных систем был использован при описании сверхпроводимости двухзонных сверхпроводников [17] и сверхтекучести электрон-позитронной плазмы [18]. В рамках этого подхода в работах [19]-[21] исследованы возможные фазовые переходы со скалярными и тензорными ПП, найдены энергетические спектры квазичастиц и температуры фазовых переходов в сверхтекучей ядерной материи.

В данной работе изучено состояние равновесия и сформулированы свойства симметрии растворов ферми-жидкостей с векторным ПП. Введены дополнительные термодинамические параметры на основе векторного ПП, получен второй закон термодинамики и найдены плотности потоков аддитивных интегралов движения в терминах плотности термодинамического потенциала в состоянии локального равновесия. Дан вывод уравнений идеальной гидродинамики без использования конкретной динамической симметрии функционала энергии и получены спектры собственных колебаний. Проведено сравнение полученных уравнений с известными уравнениями гидродинамики растворов сверхтекучих жидкостей, описываемых скалярным и тензорным ПП. Показано, что в рассматриваемых растворах ферми-жидкостей возникают эффекты увлечения, связанные с влиянием сверхтекучего движения одной компоненты на движение другой. Кроме того, имеют место эффекты увлечения нового типа, которые проявляются во взаимном влиянии спиновых и орбитальных степеней свободы. Изучено влияние этих эффектов на спектры коллективных возбуждений рассматриваемой системы.

\section{2. ПАРАМЕТРЫ ПОРЯДКА В КВАНТОВЫХ ФЕРМИ-ЖИДКОСТЯХ И ИХ РАСТВОРАХ}

При построении термодинамики и получении уравнений гидродинамики квантовых систем со спонтанно нарушенной симметрией полный набор параметров сокрашенного описания содержит, кроме плотностей аддитивных интегралов движения, также дополнительные термодинамические величины, связанные с нарушенной симметрией и определяемые в терминах ПП. Поэтому возникает необходимость изучения влияния структуры возможных ПП на термодинамику, уравнения гидродинамики и спектры коллективных возбуждений в каждом конкретном случае нарушения симметрии состояния равновесия.

В случае однокомпонентной ферми-жидкости с синглетным спариванием скалярный оператор ПП имеет вид

$$
\hat{\Delta}(\mathbf{x})=\frac{i}{2} \psi(\mathbf{x}) \sigma_{2} \psi(\mathbf{x})
$$

Случай триплетного спаривания описьвается тензорным ПП [3], [4], [22]

$$
\hat{\Delta}_{\alpha k}(\mathbf{x})=\left(\nabla_{k} \psi(\mathbf{x})\right) \sigma_{2} \sigma_{\alpha} \psi(\mathbf{x})-\psi(\mathbf{x}) \sigma_{2} \sigma_{\alpha} \nabla_{k} \psi(\mathbf{x}),
$$


где $\sigma_{\alpha}$ - матрицы Паули, $\psi, \psi^{+}$- полевые ферми-операторы.

Рассмотрим теперь раствор двух ферми-жидкостей. Здесь возможны новые типы ПП по сравнению с однокомпонентным случаем. Помимо “скалярных"

$$
\hat{\Delta}_{a}(\mathbf{x})=\frac{i}{2} \psi_{a}(\mathbf{x}) \sigma_{2} \psi_{a}(\mathbf{x}), \quad a=1,2
$$

и “тензорных"

$$
\hat{\Delta}_{a, \alpha k}(\mathbf{x})=\left(\nabla_{k} \psi_{a}(\mathbf{x})\right) \sigma_{2} \sigma_{\alpha} \psi_{a}(\mathbf{x})-\psi_{a}(\mathbf{x}) \sigma_{2} \sigma_{\alpha} \nabla_{k} \psi_{a}(\mathbf{x})
$$

ПП, описьвающих каждую компоненту в отдельности, могут быть введены также “векторные" ПП двух типов

$$
\begin{aligned}
\hat{\Delta}_{\alpha}(\mathbf{x}) & =\psi_{1}(\mathbf{x}) \sigma_{2} \sigma_{\alpha} \psi_{2}(\mathbf{x}), \\
\hat{\Delta}_{k}(\mathbf{x}) & =\frac{i}{2}\left[\left(\nabla_{k} \psi_{1}(\mathbf{x})\right) \sigma_{2} \psi_{2}(\mathbf{x})-\psi_{1}(\mathbf{x}) \sigma_{2} \nabla_{k} \psi_{2}(\mathbf{x})\right],
\end{aligned}
$$

которые строятся из полевых операторов обеих компонент раствора. Векторный ПП (4) описывает пространственно-анизотропные жидкокристаллические и изотропные в спиновом пространстве сверхтекучие состояния. ПП (3) описывает анизотропные в спиновом и изотропные в конфигурационном пространствах сверхтекучие состояния.

В этой работе мы рассмотрим состояния раствора сверхтекучих ферми-жидкостей, описываемые только ПП типа (3), и обсудим возникаюшие отличия в термодинамике и уравнениях гидродинамики от состояний растворов ферми-жидкостей со скалярньм (1) и тензорным (2) ПП.

Используя представление операторов плотностей аддитивных интегралов движения в терминах ферми-операторов рождения-уничтожения и коммутационные соотношения

$$
\begin{gathered}
\left\{\psi_{a \mu}(\mathbf{x}), \psi_{a \nu}^{+}\left(\mathbf{x}^{\prime}\right)\right\}=\delta_{\mu \nu} \delta\left(\mathbf{x}-\mathbf{x}^{\prime}\right), \quad\left\{\psi_{a \mu}(\mathbf{x}), \psi_{a \nu}\left(\mathbf{x}^{\prime}\right)\right\}=0 \\
{\left[\psi_{1 \mu}(\mathbf{x}), \psi_{2 \mu}^{+}\left(\mathbf{x}^{\prime}\right)\right]=\left[\psi_{1 \mu}(\mathbf{x}), \psi_{2 \mu}\left(\mathbf{x}^{\prime}\right)\right]=0, \quad a=1,2}
\end{gathered}
$$

легко видеть, что для оператора ПП (3) справедливы равенства

$$
\begin{aligned}
& {\left[\hat{s}_{\alpha}(\mathbf{x}), \hat{\Delta}_{\beta}\left(\mathbf{x}^{\prime}\right)\right]=i \varepsilon_{\alpha \beta \gamma} \hat{\Delta}_{\gamma}(\mathbf{x}) \delta\left(\mathbf{x}-\mathbf{x}^{\prime}\right)} \\
& {\left[\hat{n}_{1}(\mathbf{x}), \hat{\Delta}_{\alpha}\left(\mathbf{x}^{\prime}\right)\right]=\left[\hat{n}_{2}(\mathbf{x}), \hat{\Delta}_{\alpha}\left(\mathbf{x}^{\prime}\right)\right]=-\hat{\Delta}_{\alpha}(\mathbf{x}) \delta\left(\mathbf{x}-\mathbf{x}^{\prime}\right),} \\
& {\left[\hat{\pi}_{k}(\mathbf{x}), \hat{\Delta}_{\alpha}\left(\mathbf{x}^{\prime}\right)\right]=-i \hat{\Delta}_{\alpha}(\mathbf{x}) \nabla_{k} \delta\left(\mathbf{x}-\mathbf{x}^{\prime}\right) .}
\end{aligned}
$$

Формулы (6) позволяют найти законы преобразования векторного ПП при локальных преобразованиях, где генераторами являются плотности аддитивных интегралов движения. 
При локальных фазовых $U_{\varphi a} \equiv \exp \left(-i \int d^{3} x \varphi_{a}(\mathbf{x}) \hat{n}_{a}(\mathbf{x})\right), a=1,2$, и спиновых $U_{\theta} \equiv$ $\exp \left(-i \int d^{3} x \theta_{\alpha}(\mathbf{x}) \hat{s}_{\alpha}(\mathbf{x})\right)$ преобразованиях полевые ферми-операторы подчиняются соотношениям

$$
\begin{aligned}
U_{\theta}^{+} \psi_{a \mu}(\mathbf{x}) U_{\theta} & =\exp \left(\frac{i}{2} \sigma_{\alpha} \theta_{\alpha}(\mathbf{x})\right) \psi_{a \mu}(\mathbf{x}), \\
U_{\varphi a}^{+} \psi_{a \mu}(\mathbf{x}) U_{\varphi a} & =\psi_{a \mu}(\mathbf{x}) e^{-i \varphi_{a}(\mathbf{x})} \\
U_{\varphi a}^{+} \psi_{b \mu}(\mathbf{x}) U_{\varphi a} & =\psi_{b \mu}(\mathbf{x}), \quad a \neq b .
\end{aligned}
$$

При локальных преобразованиях $U_{\varphi a}, U_{\theta}$ векторньй оператор ПП (3) в силу (7) изменяется следуюшим образом:

$$
\begin{aligned}
U_{\theta}^{+} \hat{\Delta}_{\alpha}(\mathbf{x}) U_{\theta} & =a_{\alpha \beta}(\theta(\mathbf{x})) \hat{\Delta}_{\beta}(\mathbf{x}) \\
U_{\varphi a}^{+} \hat{\Delta}_{\alpha}(\mathbf{x}) U_{\varphi a} & =\hat{\Delta}_{\alpha}(\mathbf{x}) \exp \left(-2 i \varphi_{a}(\mathbf{x})\right) .
\end{aligned}
$$

В соответствии с (5) скалярные операторы ПП (1) подчиняются коммутационным соотношениям

$$
\begin{aligned}
& {\left[\hat{s}_{\alpha}(\mathbf{x}), \hat{\Delta}_{a}\left(\mathbf{x}^{\prime}\right)\right]=0, \quad\left[\hat{n}_{a}(\mathbf{x}), \hat{\Delta}_{b}\left(\mathbf{x}^{\prime}\right)\right]=-2 \delta_{a b} \hat{\Delta}_{b}(\mathbf{x}) \delta\left(\mathbf{x}-\mathbf{x}^{\prime}\right),} \\
& {\left[\hat{\pi}_{k}(\mathbf{x}), \hat{\Delta}_{a}\left(\mathbf{x}^{\prime}\right)\right]=-i \hat{\Delta}_{a}(\mathbf{x}) \nabla_{k} \delta\left(\mathbf{x}-\mathbf{x}^{\prime}\right), \quad a, b=1,2 .}
\end{aligned}
$$

При локальных спиновых и фазовых преобразованиях операторы (1) согласно (7) изменяются по законам

$$
\begin{aligned}
U_{\theta}^{+} \hat{\Delta}_{a}(\mathbf{x}) U_{\theta} & =\hat{\Delta}_{a}(\mathbf{x}), \quad U_{\varphi a}^{+} \hat{\Delta}_{b}(\mathbf{x}) U_{\varphi a}=\hat{\Delta}_{b}(\mathbf{x}), \quad a \neq b \\
U_{\varphi a}^{+} \hat{\Delta}_{a}(\mathbf{x}) U_{\varphi a} & =\hat{\Delta}_{a}(\mathbf{x}) \exp \left(-2 i \varphi_{a}(\mathbf{x})\right) .
\end{aligned}
$$

Тензорные параметры порядка (2) ввиду формул (5) удовлетворяют равенствам

$$
\begin{aligned}
i\left[\hat{s}_{\alpha}(\mathbf{x}), \hat{\Delta}_{a, \beta i}\left(\mathbf{x}^{\prime}\right)\right] & =\varepsilon_{\alpha \beta \gamma} \hat{\Delta}_{a, \gamma i}(\mathbf{x}) \delta\left(\mathbf{x}-\mathbf{x}^{\prime}\right)+\delta_{\alpha \beta} \nabla_{i}\left(\hat{\Delta}_{a}(\mathbf{x}) \delta\left(\mathbf{x}-\mathbf{x}^{\prime}\right)\right), \\
{\left[\hat{n}_{a}(\mathbf{x}), \hat{\Delta}_{b, \beta i}\left(\mathbf{x}^{\prime}\right)\right] } & =-2 \delta_{a b} \hat{\Delta}_{b, \beta i}(\mathbf{x}) \delta\left(\mathbf{x}-\mathbf{x}^{\prime}\right), \\
i\left[\hat{\pi}_{k}(\mathbf{x}), \hat{\Delta}_{a, \alpha i}\left(\mathbf{x}^{\prime}\right)\right] & =\hat{\Delta}_{a, \alpha i}(\mathbf{x}) \nabla_{k} \delta\left(\mathbf{x}-\mathbf{x}^{\prime}\right)+\nabla_{i}\left(\hat{\Delta}_{a, \alpha k}(\mathbf{x}) \delta\left(\mathbf{x}-\mathbf{x}^{\prime}\right)\right) .
\end{aligned}
$$

При локальных спиновых $U_{\theta}$ и фазовых $U_{\varphi 1}, U_{\varphi 2}$ преобразованиях с учетом $(7)$ тензорные операторы ПП (2) изменяются по законам

$$
\begin{aligned}
U_{\theta}^{+} \hat{\Delta}_{a, \alpha i}(\mathbf{x}) U_{\theta} & =a_{\alpha \beta}(\mathbf{x}) \hat{\Delta}_{a, \beta i}(\mathbf{x})-\underline{\omega}_{\alpha i}(\mathbf{x}) \hat{\Delta}_{a}(\mathbf{x}), \\
U_{\varphi a}^{+} \hat{\Delta}_{a, \alpha i}(\mathbf{x}) U_{\varphi a} & =\hat{\Delta}_{a, \alpha i}(\mathbf{x}) \exp \left(-2 i \varphi_{a}(\mathbf{x})\right) \\
U_{\varphi a}^{+} \hat{\Delta}_{b, \alpha i}(\mathbf{x}) U_{\varphi a} & =\hat{\Delta}_{b, \alpha i}(\mathbf{x}), \quad a \neq b .
\end{aligned}
$$

Здесь ортогональная матрица поворота $a_{\alpha \beta}(\theta)$ связана с локальным углом поворота $\theta_{\alpha}$ соотношением $a_{\alpha \beta}(\theta)=(\exp (-\varepsilon \theta))_{\alpha \beta}$, где $(\varepsilon \theta)_{\alpha \beta}=\varepsilon_{\alpha \beta \gamma} \theta_{\gamma}$. В терминах матрицы поворота правая форма Картана $\underline{\omega}_{\alpha k}[23]$ определяется равенством

$$
\underline{\omega}_{\alpha k}(a)=\frac{1}{2} \varepsilon_{\alpha \beta \gamma}\left(a \nabla_{k} \tilde{a}\right)_{\gamma \beta} .
$$


Преобразования произвольных деформаций задаются унитарным оператором

$$
U_{\eta} \equiv \exp \left(-i \int d^{3} x \eta_{i}(\mathbf{x}) \hat{\pi}_{i}(\mathbf{x})\right)
$$

где $\eta_{i}(\mathbf{x})$ - параметр преобразования. При преобразовании (13) операторы $\psi_{a}(\mathbf{x})$ изменяются согласно законам

$$
U_{\eta}^{+} \psi_{a}(\mathbf{x}) U_{\eta}=\alpha^{\frac{1}{2}}(\mathbf{x}) \psi_{a}\left(\mathbf{x}^{\prime}(\mathbf{x})\right), \quad a=1,2
$$

где вектор $x_{i}^{\prime}(\mathbf{x}) \equiv x_{i}-u_{i}(\mathbf{x}) \quad\left(u_{i}(\mathbf{x})\right.$ - вектор смешения) является функционалом параметра преобразования $\eta_{i}(\mathbf{x})$ и переходит в $x_{i}$ при $\eta_{i}=0, \alpha(\mathbf{x})=|\operatorname{det}(\hat{\alpha}(\mathbf{x}))|, \alpha_{i k}(\mathbf{x})=$ $\partial x_{i}^{\prime}(\mathbf{x}) / \partial x_{k}$. Тогда операторы ПП типа (1)-(3) преобразуются по законам

$$
\begin{aligned}
U_{\eta}^{+} \hat{\Delta}_{a}(\mathbf{x}) U_{\eta} & =\alpha(\mathbf{x}) \hat{\Delta}_{a}\left(\mathbf{x}^{\prime}(\mathbf{x})\right), \quad U_{\eta}^{+} \hat{\Delta}_{\alpha}(\mathbf{x}) U_{\eta}=\alpha(\mathbf{x}) \hat{\Delta}_{\alpha}\left(\mathbf{x}^{\prime}(\mathbf{x})\right) \\
U_{\eta}^{+} \hat{\Delta}_{a i}(\mathbf{x}) U_{\eta} & =\alpha(\mathbf{x}) \alpha_{k i}(\mathbf{x}) \hat{\Delta}_{a k}\left(\mathbf{x}^{\prime}(\mathbf{x})\right), \quad a=1,2
\end{aligned}
$$

Полученные в этом разделе соотношения необходимы при построении термодинамики, нахождении плотностей потоков аддитивных интегралов движения в терминах термодинамического потенциала и выводе уравнений гидродинамики.

\section{3. ОПИСАНИЕ РАВНОВЕСНОГО СОСТОЯНИЯ}

В соответствии с обшим ферми-жидкостным подходом энергия системы представляет собой функционал статистического оператора

$$
E=E(\hat{f}) \equiv \int d^{3} x e(\mathbf{x}, \hat{f})
$$

где $e(\mathbf{x}, \hat{f})$ - плотность функционала энергии системы, $\hat{f}$ - статистический оператор. Операторы энергии $\hat{\varepsilon}(\hat{f})$ и плотности энергии квазичастиц $\hat{\varepsilon}(\mathbf{x}, \hat{f})$ задаются соотношением

$$
\hat{\varepsilon}(\hat{f})=\frac{\delta E}{\delta \hat{f}}=\int d^{3} x \hat{\varepsilon}(\mathbf{x}, \hat{f}) .
$$

Дифференциальные законы сохранения для плотностей аддитивных интегралов движения $\zeta_{r}$ можно записать в виде [13]

$$
\dot{\zeta}_{r}(\mathbf{x}, \hat{f})=-\nabla_{k} \zeta_{r k}(\mathbf{x}, \hat{f})
$$

где $\zeta_{r}(\mathbf{x}, \hat{f})=\operatorname{Sp} \hat{f} \hat{\zeta}_{r}(\mathbf{x}) \quad(r=0, i, \alpha, a ; a=1,2)$ и $\zeta_{r k}(\mathbf{x}, \hat{f})=\operatorname{Sp} \hat{f} \hat{\zeta}_{r k}(\mathbf{x})-$ плотности адлитивных интегралов движения и соответствующие им потоки $\left(\hat{\zeta}_{r}(\mathbf{x}, \hat{f})=\{\hat{\varepsilon}(\mathbf{x}, \hat{f})\right.$,

6 Теоретическая и математическая физика, т. 127, № 2, 2001 г. 
$\left.\hat{\pi}_{i}(\mathbf{x}), \hat{s}_{\alpha}(\mathbf{x}), \hat{n}_{a}(\mathbf{x})\right\}$ - операторы плотностей). Операторы $\hat{\zeta}_{r k}(\mathbf{x})$ в соответствии с [13] можно представить в терминах операторов $\hat{\zeta}_{r}(\mathbf{x})$,

$$
\begin{aligned}
\hat{\zeta}_{0 k}(\mathbf{x}, \hat{f})= & \frac{i}{2} \int d^{3} x^{\prime} x_{k}^{\prime} \int_{0}^{1} d \lambda\left[\hat{\zeta}_{0}\left(\mathbf{x}-(1-\lambda) \mathbf{x}^{\prime}, \hat{f}\right), \hat{\zeta}_{0}\left(\mathbf{x}+\lambda \mathbf{x}^{\prime}\right)\right] \\
\hat{\zeta}_{r^{\prime} k}(\mathbf{x}, \hat{f})= & i \int d^{3} x^{\prime} x_{k}^{\prime} \int_{0}^{1} d \lambda\left[\hat{\zeta}_{0}\left(\mathbf{x}-(1-\lambda) \mathbf{x}^{\prime}, \hat{f}\right), \hat{\zeta}_{r^{\prime}}\left(\mathbf{x}+\lambda \mathbf{x}^{\prime}\right)\right], \quad r^{\prime}=\alpha, a, \\
\operatorname{Sp} \hat{f} \hat{\zeta}_{k j}(\mathbf{x}, \hat{f})= & -e(\mathbf{x}, \hat{f}) \delta_{k j}+i \int d^{3} x^{\prime} x_{k}^{\prime} \times \\
& \times \int_{0}^{1} d \lambda \operatorname{Sp} \hat{f}\left[\hat{\zeta}_{0}\left(\mathbf{x}-(1-\lambda) \mathbf{x}^{\prime}, \hat{f}\right), \hat{\zeta}_{j}\left(\mathbf{x}+\lambda \mathbf{x}^{\prime}\right)\right]
\end{aligned}
$$

Равновесньй статистический оператор нормального состояния раствора двух ферми-жидкостей $\hat{f}$ может быть найден исходя из требования максимума энтропии при фиксированных значениях аддитивных интегралов движения $\gamma_{r}$. Из условия экстремума для термодинамического потенциала $\Omega$ (варьирование производится по статистическому оператору $\left.\hat{f}_{0}\right)$

$$
\begin{gathered}
\delta \Omega\left(\hat{f}_{0}, Y\right)=0, \quad \Omega\left(\hat{f}_{0}, Y\right)=-\mathcal{S}\left(\hat{f}_{0}\right)+Y_{r} \gamma_{r}, \\
\mathcal{S}\left(\hat{f}_{0}\right)=-\operatorname{Sp}\left\{\hat{f}_{0} \ln \hat{f}_{0}+\left(1-\hat{f}_{0}\right) \ln \left(1-\hat{f}_{0}\right)\right\}
\end{gathered}
$$

приходим к уравнению самосогласования для равновесного оператора

$$
\hat{f}_{0}=\left[\exp \left(Y_{r} \hat{\gamma}_{r}(\hat{f})\right)+1\right]^{-1}
$$

Здесь $Y_{r}=\left(Y_{0}, Y_{\alpha}, Y_{k}, Y_{a}\right)$ - термодинамические силы, соответствующие величинам $\gamma_{r}=\left(E(\hat{f}), S_{\alpha}, \mathcal{P}_{k}, N_{a}\right)$. Равновесное состояние нормального раствора ферми-жидкостей характеризуется только термодинамическими параметрами $Y_{r}$. Состояние, описываемое статистическим оператором (18), обладает свойствами симметрии

$$
\left[\hat{f}_{0}, \widehat{\mathcal{P}}_{i}\right]=\left[\hat{f}_{0}, \widehat{S}_{\alpha}^{\prime}\right]=\left[\hat{f}_{0}, \hat{\varepsilon}\left(\hat{f}_{0}\right)\right]=\left[\hat{f}_{0}, \widehat{N}_{a}\right]=\left[\hat{f}_{0}, \hat{L}_{i}^{\prime}\right]=0
$$

Здесь $\widehat{S}_{\alpha}^{\prime}=\widehat{S}_{\alpha}-i \varepsilon_{\alpha \beta \gamma} Y_{\beta} \partial / \partial Y_{\gamma}, \hat{L}_{i}^{\prime}=\hat{L}_{i}-i \varepsilon_{i k l} Y_{k} \partial / \partial Y_{l}$ - обобщенные операторы спина и орбитального момента, а $\hat{L}_{i}=\int d^{3} x \varepsilon_{i j k} x_{j} \hat{\pi}_{k}(\mathbf{x})$ - орбитальный момент. Для описания сверхтекучих состояний в соответствии с концепцией квазисредних [24] определим равновесное среднее равенством

$$
\begin{aligned}
a(\mathbf{x}, \hat{f}) & =\operatorname{Sp} \hat{f} \hat{a}(\mathbf{x})=\lim _{\nu \rightarrow 0} \lim _{V \rightarrow \infty} \operatorname{Sp} \hat{f}_{\nu} \hat{a}(\mathbf{x}), \\
\hat{f}_{\nu} & =\left[\exp \left(Y_{r} \hat{\gamma}_{r}+\nu Y_{0} \widehat{G}\right)+1\right]^{-1},
\end{aligned}
$$

где $\hat{a}(\mathbf{x})$ - произвольный квазилокальный оператор. Источник $\widehat{G}$ нарушает соотношения симметрии (19) и представляет собой линейньй функционал операторов ПП

$$
\widehat{G}=\int d^{3} x\left\{g_{\alpha}(\mathbf{x}, t) \hat{\Delta}_{\alpha}(\mathbf{x})+g_{a}(\mathbf{x}, t) \hat{\Delta}_{a}(\mathbf{x})+g_{a, \alpha k}(\mathbf{x}, t) \hat{\Delta}_{a, \alpha k}(\mathbf{x})+\text { э.c. }\right\} .
$$


По повторяющимся индексам подразумевается суммирование. Конкретный вид функций $g_{a}(\mathbf{x}, t), g_{\alpha}(\mathbf{x}, t), g_{a, \alpha k}(\mathbf{x}, t)$ определяется свойствами симметрии состояния равновесия.

Рассмотрим одно из возможных состояний равновесия раствора ферми-жидкостей, обладающее свойствами симметрии [11]

$$
\begin{gathered}
{\left[\hat{f}^{\mathrm{s}}, \widehat{\mathcal{P}}_{k}-p_{a k} \widehat{N}_{a}\right]=0, \quad\left[\hat{f}^{\mathrm{s}}, \hat{\varepsilon}(\hat{f})+p_{a 0} \widehat{N}_{a}\right]=0, \quad\left[\hat{f}^{\mathrm{s}}, \widehat{S}_{\alpha}^{\prime}\right]=0} \\
{\left[\hat{f}^{\mathrm{s}}, \hat{L}_{k}^{\prime \prime}\right]=0, \quad \hat{L}_{i}^{\prime \prime}=\hat{L}_{i}^{\prime}-i \varepsilon_{i k l} p_{a k} \frac{\partial}{\partial p_{a l}}, \quad p_{20}=Y_{0}^{-1}\left(Y_{a}+Y_{k} p_{a k}\right) .}
\end{gathered}
$$

Здесь $p_{a k}$ - сверхтекучие импульсы в равновесном состоянии. Легко видеть, что вследствие коммутационных соотношений (6), (11) и свойств симметрии состояния (21) средние значения векторного и тензорных ПП в этом состоянии обрашаются в ноль (ПП (2), (3) обрашаются в ноль в силу третьего соотношения свойств симметрии (21)). Равновесное значение скалярного ПП можно представить в виде

$$
\Delta_{a}^{(\mathrm{s})}(\mathbf{x})=\Delta_{a}(\mathrm{inv}) e^{i\left(\mathbf{p}_{a} \mathbf{x}+\theta_{a}\right)}, \quad \Delta_{\alpha}^{(\mathrm{s})}=\Delta_{a, \alpha k}^{(\mathrm{s})}=0, \quad a=1,2,
$$

где индекс "s" соответствует равновесному состоянию (21). Назовем такие состояния синглетныли, т.к. они характеризуются ненулевым синглетным ПП в этом состоянии. Такого типа состояния исследовались в работах [9], [11].

Другим возможным равновесным состоянием раствора ферми-жидкостей является состояние, обладаюшее свойствами симметрии [14]

$$
\begin{gathered}
{\left[\hat{f}^{\mathrm{t}}, \widehat{\mathcal{P}}_{k}-q_{k} n_{\alpha} \widehat{S}_{\alpha}^{\prime}-p_{a k} \widehat{N}_{a}\right]=0, \quad\left[\hat{f}^{\mathrm{t}}, \hat{\varepsilon}(\hat{f})+\omega_{\alpha 0} \widehat{S}_{\alpha}^{\prime}+p_{a 0} \widehat{N}_{a}\right]=0} \\
{\left[\hat{f}^{\mathrm{t}}, \hat{L}_{k}^{\prime \prime}+a_{k \alpha}^{\prime} \widehat{S}_{\alpha}^{\prime}\right]=0, \quad \hat{L}_{i}^{\prime \prime}=\hat{L}_{i}^{\prime}-i \varepsilon_{i k l}\left(p_{a k} \frac{\partial}{\partial p_{a l}}+q_{k} \frac{\partial}{\partial q_{l}}\right)} \\
\omega_{\alpha 0}=Y_{0}^{-1}\left(Y_{\alpha}+Y_{k} q_{k} n_{\alpha}\right), \quad p_{a 0}=Y_{0}^{-1}\left(Y_{a}+Y_{k} p_{a k}\right)
\end{gathered}
$$

Здесь $n_{\alpha}=Y_{\alpha} /|Y|$ определяется из условия совместности соотношений пространственной однородности и стационарности (первое и второе соотношения из свойств $(23))$. Было найдено, что для такого состояния в силу соотношений $(6),(9)$ и свойств симметрии (23) скалярные и векторньй ПП обращаются в ноль (в силу третьего из соотношений симметрии (23)), а тензорные ПП в этом состоянии представимы в форме

$$
\Delta_{a, \alpha k}^{(\mathrm{t})}(\mathbf{x})=\Delta_{a}(\mathrm{inv}) a_{k \beta}^{\prime} a_{\beta \alpha} e^{i\left(\mathbf{p}_{a} \mathbf{x}+\theta_{a}\right)}, \quad \Delta_{a}^{(\mathrm{t})}=\Delta_{\alpha}^{(\mathrm{t})}=0, \quad a=1,2 .
$$

Индекс “t” соответствует равновесному состоянию (23). Его по аналогии можно назвать триплетным. Оно описывает класс возможных равновесных состояний типа В-фазы сверхтекучего Не-3, в котором отличны от нуля равновесные значения спина и вектора магнитной спирали в растворе двух квантовых ферми-жидкостей.

При формулировке свойств симметрии состояния равновесия раствора ферми-жидкостей с векторным ПП мы исходим из того, что выписанный набор свойств симметрии образует замкнутую операторную алгебру, и из требования обрашения в ноль в этом 
состоянии скалярных и тензорных ПП. При этом векторный ПП (3) отличен от нуля. Эти свойства симметрии имеют вид

$$
\begin{gathered}
{\left[\hat{f}^{\mathrm{v}}, \widehat{\mathcal{P}}_{k}-q_{k} n_{\alpha} \widehat{S}_{\alpha}^{\prime}-p_{a k} \widehat{N}_{a}\right]=0, \quad\left[\hat{f}^{\mathrm{v}}, \hat{\varepsilon}(\hat{f})+\omega_{\alpha 0} \widehat{S}_{\alpha}^{\prime}+p_{a 0} \widehat{N}_{a}\right]=0,} \\
{\left[\hat{f}^{\mathrm{v}}, \underline{d}_{\alpha} \widehat{S}_{\alpha}^{\prime}-m_{\mathrm{s}}\left(\widehat{N}_{1}+\widehat{N}_{2}\right)\right]=0, \quad\left[\hat{f}^{\mathrm{v}}, \hat{L}_{k}^{\prime \prime}+\varrho_{k}\left(\widehat{N}_{1}-\widehat{N}_{2}\right)\right]=0,} \\
\hat{L}_{i}^{\prime \prime}=\hat{L}_{i}^{\prime}-i \varepsilon_{i k l}\left(p_{a k} \frac{\partial}{\partial p_{a l}}+q_{k} \frac{\partial}{\partial q_{l}}\right), \quad \omega_{\alpha 0}=Y_{0}^{-1}\left(Y_{\alpha}+Y_{k} q_{k} n_{\alpha}\right) .
\end{gathered}
$$

Здесь $n_{\alpha}=Y_{\alpha} /|Y|$ определяется таким же образом, как в случае (23), $q_{i}$ - вектор магнитной спирали, $\underline{d}_{\alpha}$ - вектор магнитной анизотропии, $m_{\mathrm{s}}-$ некоторая константа и $\varrho_{i}-$ некоторый вектор, которые мы определим ниже.

Воспользовавшись соотношениями (9), (11) и свойствами симметрии (25), получим, что скалярные и тензорные ПП обрашаются в ноль (ПП (1), (2) обрашаются в ноль в силу четвертого из соотношений симметрии (25)), а векторньй ПП в этом состоянии отличен от нуля и имеет вид

$$
\begin{gathered}
\Delta_{\alpha}^{(\mathrm{v})}(\mathbf{x})=\left(\Delta_{I} e_{\alpha}(\mathbf{x})+i \Delta_{I I} f_{\alpha}(\mathbf{x})\right) e^{i\left(\left(\mathbf{p}_{1}+\mathbf{p}_{2}\right) \mathbf{x}+\theta^{\prime}\right)}, \\
\Delta_{a}^{(\mathrm{v})}=0, \quad \Delta_{a, \alpha k}^{(\mathrm{v})}=0, \quad a=1,2, \quad e^{2}=f^{2}=1, \quad \text { ef }=0, \quad \mathbf{d}=\mathbf{e} \times \mathbf{f} .
\end{gathered}
$$

Выражение для $\Delta_{\alpha}^{(\mathrm{v})}(\mathbf{x})$ непосредственно вытекает из первого соотношения симметрии (25). Здесь индекс "v" соответствует равновесному состоянию (25). Рассмотренное состояние будем назьвать векторным.

Исходя из групповых свойств симметрии (25) найдем выражение для $m_{\mathrm{s}}$. Используя формулы (6), (25), (26), получим $2 m_{\mathrm{S}} \Delta_{I}+\Delta_{I I}=0, \Delta_{I}+2 m_{\mathrm{s}} \Delta_{I I}=0$. Эта система имеет нетривиальные решения для $\Delta_{I}, \Delta_{I I}$ тогда и только тогда, когда $m_{\mathrm{S}}= \pm 1 / 2$.

Для того чтобы выражение для $\Delta_{\alpha}^{(\mathrm{v})}(\mathbf{x})(26)$ не обрашалось в ноль, достаточно рассмотреть свойство симметрии $\left[\hat{f}^{v}, \hat{L}_{k}^{\prime \prime}\right]=0$. В этом случае также остаются отличные от нуля значения скалярных ПП $\Delta_{a}^{(\mathrm{v})}(\mathbf{x})$. Введение дополнительного слагаемого $\varrho_{k}\left(\widehat{N}_{1}-\widehat{N}_{2}\right)$ в симметрийное соотношение обрашает в ноль последние. Определим вектор $\varrho_{i}$, воспользовавшись тождеством Якоби и требованием полноты системы свойств симметрии (25). Этот вектор в силу соотношения

$$
\left[\hat{L}_{k}^{\prime \prime}+\varrho_{k}\left(\widehat{N}_{1}-\widehat{N}_{2}\right), \hat{L}_{l}^{\prime \prime}+\varrho_{l}\left(\widehat{N}_{1}-\widehat{N}_{2}\right)\right]=i \varepsilon_{k l m}\left(\hat{L}_{m}^{\prime \prime}+\varrho_{m}\left(\widehat{N}_{1}-\widehat{N}_{2}\right)\right)
$$

имеет вид $\varrho_{i}=\left[\mathbf{p}_{2} \times \mathbf{p}_{1}\right]_{i} /\left|\left[\mathbf{p}_{2} \times \mathbf{p}_{1}\right]\right|$.

Учитывая (6), (9), (11), (25), найдем функции $g$ в этом состоянии

$$
\begin{aligned}
g_{a}(\mathbf{x}, t) & =\exp \left(-i \varphi_{a}(\mathbf{x}, t)\right), \quad a=1,2 \\
g_{a, \alpha k}(\mathbf{x}, t) & =a_{k \beta}^{\prime} a_{\beta \alpha}(\theta(\mathbf{x}, t)) \exp \left(-2 i \varphi_{a}(\mathbf{x})\right) \\
g_{\alpha}(\mathbf{x}, t) & =\left(e_{\alpha}(\mathbf{x}, t)+2 i m_{\mathrm{s}} f_{\alpha}(\mathbf{x}, t)\right) \exp \left(-i\left(\varphi_{1}(\mathbf{x}, t)+\varphi_{2}(\mathbf{x}, t)\right)\right), \\
e_{\alpha}(\mathbf{x}, t) & =\underline{e}_{\beta} a_{\beta \alpha}(\mathbf{x}, t), \quad f_{\alpha}(\mathbf{x}, t)=\underline{f}_{\beta} a_{\beta \alpha}(\mathbf{x}, t), \\
\theta_{\alpha}(\mathbf{x}, t) & =n_{\alpha}\left(\mathbf{q} \mathbf{x}-q_{0} t\right)+\theta_{\alpha}^{0}, \quad \varphi_{a}(\mathbf{x}, t)=\mathbf{p}_{a} \mathbf{x}-p_{a 0} t+\varphi_{a 0} .
\end{aligned}
$$


Состояние равновесия типа (25) характеризуется следующими дополнительными термодинамическими параметрами: сверхтекучими фазами и импульсами, вектором магнитной анизотропии, вектором магнитной спирали, ортогональной матрищей однородного спинового поворота.

\section{4. ЛОКАЛЬНО-РАВНОВЕСНЫЕ СОСТОЯНИЯ}

Статистический оператор, описывающий локально-равновесные вырожденные состояния, определим равенством

$$
\begin{aligned}
& \hat{f}\left\{Y(\mathbf{x}), a(\mathbf{x}), \varphi_{a}(\mathbf{x})\right\}=\left[\operatorname { e x p } \int d ^ { 3 } x \left(Y_{r}(\mathbf{x}) \hat{\zeta}_{r}(\mathbf{x}, \hat{f})+\nu Y_{0}(\mathbf{x}) U_{\varphi 1}^{+} U_{\varphi 2}^{+} U_{\theta}^{+} \times\right.\right. \\
& \left.\left.\quad \times\left(g_{a}(\mathbf{x}) \hat{\Delta}_{a}(\mathbf{x})+g_{a, \alpha k}(\mathbf{x}) \hat{\Delta}_{a, \alpha k}(\mathbf{x})+g_{\alpha}(\mathbf{x}) \hat{\Delta}_{\alpha}(\mathbf{x})+\text { э.c. }\right) U_{\theta} U_{\varphi 2} U_{\varphi 1}\right)+1\right]^{-1},
\end{aligned}
$$

где $g_{a}(\mathbf{x}), g_{\alpha}(\mathbf{x}), g_{a, \alpha k}(\mathbf{x})$ - некоторые с-числовые функции. В этом состоянии термодинамические параметры $Y(\mathbf{x})$, сверхтекучие фазы $\varphi_{a}(\mathbf{x})(a=1,2)$ и ортогональная матрица $a(\mathbf{x})$ являются произвольными функциями координат. В состоянии полного равновесия термодинамические силы $Y(\mathbf{x})=Y$ однородны, а структура сверхтекучих фаз $\varphi_{a}(\mathbf{x})$ и ортогональной матришы $a_{\alpha \beta}(\mathbf{x})$ определяются формулами $(27)$.

Определим локально-равновесный термодинамический потенциал равенством

$$
\Omega\left(\hat{f}\left(Y, a, \varphi_{a}\right), Y\right)=-\mathcal{S}(\hat{f})+\int d^{3} x Y_{r}(\mathbf{x}) \operatorname{Sp} \hat{f} \hat{\zeta}_{r}(\mathbf{x}) .
$$

Здесь $Y_{r}(\mathbf{x})$ - множители Лагранжа, соответствуюшие величинам $\zeta_{r}(\mathbf{x})=\operatorname{Sp} \hat{f} \hat{\zeta}_{r}(\mathbf{x})$. Локально-равновесньй термодинамический потенциал $\Omega$ является функционалом термодинамических параметров $\underline{Y}_{r}=\left\{Y_{0}, \underline{Y}_{\alpha}=a_{\alpha \beta} Y_{\beta}, Y_{i}, Y_{a}\right\}$, правой формы Картана $\underline{\omega}_{\alpha k}\left(\mathbf{x}^{\prime}\right)$ и сверхтекучих импульсов $\mathbf{p}_{a}=\nabla \theta_{a}$,

$$
\Omega=\Omega\left(\underline{Y}\left(\mathbf{x}^{\prime}\right), \underline{\omega}_{k}\left(\mathbf{x}^{\prime}\right), \mathbf{p}_{a}\left(\mathbf{x}^{\prime}\right)\right)=\int d^{3} x \omega\left(\mathbf{x}, \underline{Y}\left(\mathbf{x}^{\prime}\right), \underline{\omega}_{k}\left(\mathbf{x}^{\prime}\right), \varphi_{a}\left(\mathbf{x}^{\prime}\right)\right),
$$

где $\omega$-плотность термодинамического потенциала. Варьируя (30) по термодинамическим параметрам, получим

$$
\delta \omega=\frac{\partial \omega}{\partial \underline{Y}_{r}} \delta \underline{Y}_{r}+\frac{\partial \omega}{\partial \underline{\omega}_{\alpha k}} \delta \underline{\omega}_{\alpha k}+\frac{\partial \omega}{\partial p_{a k}} \delta p_{a k} .
$$

Получим выражения для плотностей потоков числа частищ каждого сорта. Рассмотрим потенциал (29), который связан с группой произвольных фазовых преобразований. Его вариацию запишем в виде

$$
\delta_{\varphi a} \Omega=-i \int d^{3} x d^{3} x^{\prime} \delta \varphi_{a}\left(\mathbf{x}^{\prime}\right) \operatorname{Sp} \hat{f}\left[\hat{n}_{a}\left(\mathbf{x}^{\prime}\right), Y_{r}(\mathbf{x}) \hat{\zeta}_{r}(\mathbf{x})\right], \quad a=1,2 .
$$


Используя коммутационные соотношения для аддитивных интегралов движения, получим

$$
\delta_{\varphi a} \Omega=\int d^{3} x\left(Y_{0}(\mathbf{x}) i_{a k}(\mathbf{x})+Y_{k}(\mathbf{x}) \frac{\partial \omega}{\partial Y_{a}}\right) \nabla_{k} \delta \varphi_{a}(\mathbf{x}) .
$$

С другой стороны, можно записать, что

$$
\delta_{\varphi a} \Omega=\int d^{3} x \delta_{\varphi a} \omega(\mathbf{x})=\int d^{3} x \frac{\partial \omega}{\partial p_{a k}} \nabla_{k} \delta \varphi_{a}(\mathbf{x})
$$

Сравнивая два последних выражения, найдем плотности потока числа частиц каждого сорта

$$
j_{a k}=\frac{1}{Y_{0}} \frac{\delta \omega}{\delta p_{a k}}-\frac{Y_{k}}{Y_{0}} \frac{\delta \omega}{\delta Y_{a}} .
$$

Теперь найдем выражение для плотности потока спина. Вариация потенциала (29), связанная со спиновыми врашениями, может быть представлена в виде

$$
\delta_{\theta} \Omega=-i \int d^{3} x d^{3} x^{\prime} \delta R_{\alpha}\left(\mathbf{x}^{\prime}\right) \operatorname{Sp} \hat{f}\left[\hat{s}_{\alpha}\left(\mathbf{x}^{\prime}\right), Y_{r}(\mathbf{x}) \hat{\zeta}_{r}(\mathbf{x})\right], \quad \delta R_{\alpha}=\frac{1}{2} \varepsilon_{\alpha \beta \gamma} a_{\rho \gamma} \delta a_{\rho \beta} .
$$

Снова воспользуемся коммутационными соотношениями для аддитивных интегралов движения и получим

$$
\begin{aligned}
\delta_{\theta} \Omega & =\int d^{3} x\left\{-\left(Y_{0}(\mathbf{x}) j_{\alpha k}(\mathbf{x})+Y_{k}(\mathbf{x}) \frac{\partial \omega}{\partial Y_{\alpha}}\right) \nabla_{k} \delta R_{\alpha}-\varepsilon_{\alpha \beta \gamma} Y_{\beta} \frac{\partial \omega}{\partial Y_{\gamma}}\right\}= \\
& =-\int d^{3} x\left(Y_{0}(\mathbf{x}) \underline{j}_{\alpha k}(\mathbf{x})+Y_{k}(\mathbf{x}) \frac{\partial \omega}{\partial \underline{Y}_{\alpha}}\right) \delta \underline{\omega}_{\alpha k} .
\end{aligned}
$$

С другой стороны, имеем

$$
\delta_{\theta} \Omega=\int d^{3} x \delta_{\theta} \omega(\mathbf{x})=\int d^{3} x \frac{\partial \omega}{\partial \underline{\omega}_{\alpha k}} \delta \underline{\omega}_{\alpha k}(\mathbf{x}) .
$$

Сравнивая последние выражения, найдем, что

$$
\underline{j}_{\alpha k}=a_{\alpha \beta} j_{\beta k}=\frac{1}{Y_{0}} \frac{\delta \omega}{\delta \underline{\omega}_{\alpha k}}-\frac{Y_{k}}{Y_{0}} \frac{\delta \omega}{\delta \underline{Y}_{\alpha}} .
$$

Подставляя (32), (33) в выражение (31), получим второй закон термодинамики для локально-равновесного случая

$$
\begin{gathered}
\delta \omega=\underline{\zeta}_{r} \delta \underline{Y}_{r}+\left(Y_{0} \underline{j}_{\alpha k}+Y_{k} \underline{s}_{\alpha}\right) \delta \underline{\omega}_{\alpha k}+\left(Y_{0} j_{a k}+Y_{k} n_{a}\right) \delta p_{a k} \\
\underline{\zeta}_{r}=\operatorname{Sp} \underline{\hat{f}} \zeta_{r}=\left\{\varepsilon, \pi_{i}, \underline{s}_{\alpha}=a_{\alpha \beta} s_{\beta}, n_{a}\right\}, \quad \underline{j}_{\alpha k}=a_{\alpha \beta} j_{\beta k} .
\end{gathered}
$$

Найдем теперь выражение для тензора натяжений. С этой целью рассмотрим модифицированный потенциал, связанный с группой произвольных деформаций $\eta_{i}(\mathbf{x})$

$$
\Omega\left(f\left(Y, a, \varphi_{a}, \eta\right), Y\right) \equiv-\mathcal{S}(\hat{f})+\int d^{3} x Y_{r}(\mathbf{x}) \operatorname{Sp} U_{\eta} \hat{f} U_{\eta}^{+} \hat{\zeta}_{r}(\mathbf{x})
$$


Его вариация записьвается в виде

$$
\delta_{\eta} \Omega=-i \int d^{3} x d^{3} x^{\prime} \delta \eta_{i}\left(\mathbf{x}^{\prime}\right) \operatorname{Sp} \hat{f}\left[\hat{\pi}_{i}\left(\mathbf{x}^{\prime}\right), Y_{r}(\mathbf{x}) \hat{\zeta}_{r}(\mathbf{x})\right]
$$

Используя коммутационные соотношения для аддитивных интегралов движения, получим

$$
\delta_{\eta} \Omega=\int d^{3} x \delta \eta_{i}(\mathbf{x})\left\{-\nabla_{k}\left(Y_{0} t_{i k}+\frac{\partial \omega Y_{k}}{\partial Y_{i}}\right)(\mathbf{x})+\frac{\partial \omega}{\partial p_{a k}} \nabla_{i} p_{a k}(\mathbf{x})+\frac{\partial \omega}{\partial \underline{\omega}_{\alpha k}} \nabla_{i} \underline{\omega}_{\alpha k}(\mathbf{x})\right\} .
$$

С другой стороны, согласно явному виду сверхтекучих импульсов $p_{a k} \equiv p_{a k}\left(U_{\eta} \hat{f} U_{\eta}^{+}\right)$ и формы Картана $\underline{\omega}_{\alpha k} \equiv \underline{\omega}_{\alpha k}\left(U_{\eta} \hat{f} U_{\eta}^{+}\right)$, найдем их трансформационные свойства при бесконечно малых преобразованиях деформаций

$$
\delta p_{a k}=-\nabla_{k} \delta \eta_{i} p_{a i}-\nabla_{i} p_{a k} \delta \eta_{i}, \quad \delta \underline{\omega}_{\alpha k}=-\nabla_{k} \delta \eta_{i} \underline{\omega}_{\alpha i}+\nabla_{i} \underline{\omega}_{\alpha k} \delta \eta_{i}
$$

Отсюда в силу (36) получим вариацию

$$
\begin{aligned}
\delta_{\eta} \Omega & =\int d^{3} x \delta_{\eta} \omega(\mathbf{x})=\int d^{3} x\left\{\frac{\partial \omega}{\partial p_{a k}} \delta_{\eta} p_{a k}(\mathbf{x})+\frac{\partial \omega}{\partial \underline{\omega}_{\alpha k}} \delta_{\eta} \underline{\omega}_{\alpha k}(\mathbf{x})\right\}= \\
& =\int d^{3} x\left\{\frac{\partial \omega}{\partial p_{a k}}\left(p_{a i} \nabla_{k} \delta \eta_{i}+\nabla_{i} p_{k} \delta \eta_{i}\right)+\frac{\partial \omega}{\partial \underline{\omega}_{\alpha k}}\left(\underline{\omega}_{\alpha i} \nabla_{k} \delta \eta_{i}+\nabla_{i} \underline{\omega}_{\alpha k} \delta \eta_{i}\right)\right\}
\end{aligned}
$$

Сравнив два последних выражения, найдем тензор натяжений

$$
t_{i k}=-\frac{\partial}{\partial Y_{i}} \frac{\omega Y_{k}}{Y_{0}}+\frac{p_{a i}}{Y_{0}} \frac{\partial \omega}{\partial p_{a k}}+\frac{\underline{\omega}_{\alpha i}}{Y_{0}} \frac{\partial \omega}{\partial \underline{\omega}_{\alpha k}} .
$$

Выражение для плотности потока энергии получим (следуя подходу работы [14]), используя соотношение

$$
\underline{Y}_{r}\left(Y_{0} \underline{\zeta}_{r k}+Y_{k} \underline{\zeta}_{r}\right)=0
$$

и выражения для остальных потоков $(32),(33),(36)$. Плотность потока энергии имеет Вид

$$
w_{k}=-\frac{\partial}{\partial Y_{0}} \frac{\omega Y_{k}}{Y_{0}}-\frac{p_{a 0}}{Y_{0}} \frac{\partial \omega}{\partial p_{a k}}+\frac{\underline{\omega}_{\alpha 0}}{Y_{0}} \frac{\partial \omega}{\partial \underline{\omega}_{\alpha k}} .
$$

В итоге все локально-равновесные плотности потоков в рассматриваемом состоянии можно представить в компактном виде

$$
\underline{\zeta}_{r k}=-\frac{\partial}{\partial \underline{Y}_{r}} \frac{\omega Y_{k}}{Y_{0}}+\frac{\partial p_{a 0}}{\partial \underline{Y}_{r}} \frac{\partial \omega}{\partial p_{a k}}+\frac{\partial \underline{\omega}_{\alpha 0}}{\partial \underline{Y}_{r}} \frac{\partial \omega}{\partial \underline{\omega}_{\alpha k}}
$$

Перейдем от переменных $a_{\alpha \beta}, \varphi_{a}, \underline{\omega}_{\alpha k}$ к новым переменным, также выражающимся в терминах ПП. Вместо матрицы поворота $a_{\alpha \beta}$ и связанной с ней формы Картана $\omega_{\alpha k}$ введем единичный вектор $d_{\alpha}$, имеюший смысл вектора магнитной анизотропии, вектор 
магнитной спирали $q_{i}$ и поперечную часть формы Картана $z_{\alpha k}$. Все эти новые переменные могут быть выражены через векторный ПП согласно формулам

$$
\begin{aligned}
d_{\alpha}(\mathbf{x}) & =[\mathbf{e}(\mathbf{x}) \mathbf{f}(\mathbf{x})]_{\alpha}=\left\{1-\left(\boldsymbol{\xi}_{2}(\mathbf{x}) \boldsymbol{\xi}_{1}(\mathbf{x})\right)^{2}\right\}^{-\frac{1}{2}}\left[\boldsymbol{\xi}_{2}(\mathbf{x}) \boldsymbol{\xi}_{1}(\mathbf{x})\right]_{\alpha}, \\
q_{k}(\mathbf{x}) & =\frac{1}{2}\left\{1-\left(\boldsymbol{\xi}_{2}(\mathbf{x}) \boldsymbol{\xi}_{1}(\mathbf{x})\right)^{2}\right\}^{-\frac{1}{2}}\left(\boldsymbol{\xi}_{2}(\mathbf{x}) \nabla_{k} \boldsymbol{\xi}_{1}(\mathbf{x})-\boldsymbol{\xi}_{1}(\mathbf{x}) \nabla_{k} \boldsymbol{\xi}_{2}(\mathbf{x})\right), \\
z_{\alpha k} & =\left[\left(\nabla_{k} \mathbf{d}(\mathbf{x})\right) \times \mathbf{d}(\mathbf{x})\right]_{\alpha}, \quad \xi_{1 \alpha}(\mathbf{x})=\frac{\Delta_{1 \alpha}(\mathbf{x})}{\Delta_{1}(\mathbf{x})}, \quad \xi_{2 \alpha}(\mathbf{x})=\frac{\Delta_{2 \alpha}(\mathbf{x})}{\Delta_{2}(\mathbf{x})} .
\end{aligned}
$$

В терминах этих величин второй закон термодинамики и выражения для плотностей потоков принимают вид

$$
\begin{gathered}
\delta \omega=\underline{\zeta}_{r} \delta \underline{Y}_{r}+\left(Y_{0} \underline{j}_{\alpha k}+Y_{k} \underline{s}_{\alpha}\right)\left(\underline{d}_{\alpha} \delta q_{k}+\delta \underline{z}_{\alpha k}\right)+\left(Y_{0} j_{a k}+Y_{k} n_{a}\right) \delta p_{a k}, \\
\underline{\zeta}_{r k}=-\frac{\partial}{\partial \underline{Y}_{r}} \frac{\omega Y_{k}}{Y_{0}}+\frac{\partial p_{a 0}}{\partial \underline{Y}_{r}} \frac{\partial \omega}{\partial p_{a k}}+\frac{\partial q_{0}}{\partial \underline{Y}_{r}} \frac{\partial \omega}{\partial q_{k}}+\frac{\partial \underline{z}_{\alpha 0}}{\partial \underline{Y}_{r}} \frac{\partial \omega}{\partial \underline{z}_{\alpha k}} .
\end{gathered}
$$

Здесь $q_{0}=Y_{0}^{-1}\left(\underline{d}_{\alpha} Y_{\alpha}+Y_{k} q_{k}\right), \underline{z}_{\alpha 0}=Y_{0}^{-1}\left(Y_{\alpha}-d_{\beta} d_{\alpha} Y_{\beta}+Y_{k} z_{\alpha k}\right)$. Как ниже будет показано, в этих терминах удобно представить влияние эффектов увлечения на термодинамику и спектры собственных колебаний.

Плотность термодинамического потенциала $\omega$ для раствора двух ферми-жидкостей зависит от следующих инвариантов, квадратичных по термодинамическим параметpaм: $Y_{0}, Y_{k}^{2}, Y_{\beta}^{2}, Y_{a}, Y_{k} p_{a k}, Y_{k} q_{k}, Y_{\beta} d_{\beta}, p_{a}^{2}, p_{a}^{2}, p_{1 k} p_{2 k}, p_{a k} q_{k}, q^{2}, z^{2}$.

Чтобы проследить взаимосвязь между полученными в этой работе выражениями и выражениями для потоков и потоками в "двухжидкостной” формулировке Ландау-Тиссы, введем физические величины

$$
\begin{gathered}
\rho_{\mathrm{n}}=-2 Y_{0} \frac{\partial \omega}{\partial \mathbf{Y}^{2}}, \quad \rho_{\mathrm{s} a}=2 \frac{m_{a}^{* 2}}{Y_{0}} \frac{\partial \omega}{\partial \mathbf{p}_{a}^{2}}, \quad \rho_{\mathrm{s} 12}=\frac{m_{1}^{*} m_{2}^{*}}{Y_{0}} \frac{\partial \omega}{\partial \mathbf{p}_{1} \mathbf{p}_{2}}, \\
\nu=\frac{\partial \omega}{\partial \mathbf{Y} \mathbf{q}}, \quad \rho-\rho_{\mathrm{n}}-\rho_{\mathrm{s} 2}-\rho_{\mathrm{s} 12}=m_{1}^{*} \frac{\partial \omega}{\partial \mathbf{Y}_{1}}, \\
\rho-\rho_{\mathrm{n}}-\rho_{\mathrm{s} 1}-\rho_{\mathrm{s} 12}=m_{2}^{*} \frac{\partial \omega}{\partial \mathbf{Y} \mathbf{p}_{2}}, \quad \kappa_{1}=\frac{\partial \omega}{\partial \mathbf{p}_{1} \mathbf{q}}, \\
\tau_{\|}=\frac{2}{Y_{0}} \frac{\partial \omega}{\partial \mathbf{q}^{2}}, \quad \tau_{\perp}=\frac{2}{Y_{0}} \frac{\partial \omega}{\partial \mathbf{z}^{2}}, \quad s_{\|}=\frac{\partial \omega}{\partial \mathbf{d Y}}, \quad \chi=2 Y_{0} \frac{\partial \omega}{\partial Y_{\alpha}^{2}}, \quad \kappa_{2}=\frac{\partial \omega}{\partial \mathbf{p}_{2} \mathbf{q}},
\end{gathered}
$$

где потенциал $\omega$ зависит только от инвариантов линейных и квадратичных по векторным термодинамическим параметрам. В выражениях (43) $\rho_{\mathrm{n}}$ - нормальная плотность раствора, $\rho_{\mathrm{s} 1}, \rho_{\mathrm{s} 2}$ - сверхтекучие плотности компонент, $m_{1}^{*}, m_{2}^{*}-$ эффективные массы компонент, $\rho_{\mathrm{S} 12}$ - "увлекаемая" сверхтекучая плотность. Величины $\tau_{\|}, \tau_{\perp}$ представляют собой продольную и поперечную по отношению к вектору магнитной анизотропии константы жесткости. Величина $s_{\|}$- продольная составляюшая спина и $\chi$ - магнитная 
восприимчивость. Физические параметры $\kappa_{1}$ и $\kappa_{2}$ описывают, как будет показано ниже, эффект спин-орбитального увлечения. Используя введенные вьше величины, все плотности потоков можно записать в виде

$$
\begin{aligned}
j_{1 i}= & \frac{1}{m_{1}^{*}}\left[\left(\rho_{\mathrm{n}}+\rho_{\mathrm{s} 2}-\rho+m_{1}^{*} n_{1}\right) v_{\mathrm{n} i}+\rho_{\mathrm{s} 1} v_{\mathrm{s} 1 i}+\rho_{\mathrm{s} 12} v_{\mathrm{s} 2 i}\right]+\kappa_{1} q_{i}, \\
j_{2 i}= & \frac{1}{m_{2}^{*}}\left[\left(\rho_{\mathrm{n}}+\rho_{\mathrm{s} 1}-\rho+m_{2}^{*} n_{2}\right) v_{\mathrm{n} i}+\rho_{\mathrm{s} 12} v_{\mathrm{s} 1 i}+\rho_{\mathrm{s} 2} v_{\mathrm{s} 2 i}\right]+\kappa_{2} q_{i}, \\
j_{\alpha i}^{\perp}= & s_{\alpha}^{\perp} v_{\mathrm{n} i}+z_{\alpha i} \tau_{\perp}, \quad j_{\| i}=\left(s_{\|}+\nu\right) v_{\mathrm{n} i}+q_{i} \tau_{\|}+m_{1}^{*} \kappa_{1} v_{\mathrm{s} 1 i}+m_{2}^{*} \kappa_{2} v_{\mathrm{s} 2 i}, \\
t_{i k}= & p \delta_{i k}+\rho_{\mathrm{n}} v_{\mathrm{n} i} v_{\mathrm{n} k}+\rho_{\mathrm{s} 1} v_{\mathrm{s} 1 i} v_{\mathrm{s} 1 k}+\rho_{\mathrm{s} 2} v_{\mathrm{s} 2 i} v_{\mathrm{s} 2 k}+\rho_{\mathrm{s} 12}\left(v_{\mathrm{s} 1 i} v_{\mathrm{s} 2 k}+v_{\mathrm{s} 2 i} v_{\mathrm{s} 1 k}\right)+ \\
& \quad+\frac{m_{1}^{*}}{Y_{0}} \kappa_{1}\left(v_{\mathrm{s} 1 i} q_{k}+v_{\mathrm{s} 1 k} q_{i}\right)+\frac{m_{2}^{*}}{Y_{0}} \kappa_{2}\left(v_{\mathrm{s} 2 i} q_{k}+v_{\mathrm{s} 2 k} q_{i}\right)+\tau_{\|} q_{i} q_{k}+\tau_{\perp} \underline{z}_{\alpha i} \underline{z}_{\alpha k} .
\end{aligned}
$$

Плотность потока энергии в этом случае можно получить, используя формулы (44) и (37). Потоки (44) выписаны с точностью до квадратичных по термодинамическим параметрам слагаемых. Так как векторы $v_{\mathrm{s} a k}, q_{k}$ в равновесии мы считаем малыми, то слагаемыми более высоких порядков можно пренебречь. В формулах (44) $j_{\| i}=d_{\alpha} j_{\alpha i}$ - продольная часть плотности потока спина, а $j_{\alpha i}^{\perp}=\left(\delta_{\alpha \beta}-d_{\alpha} d_{\beta}\right) j_{\beta i}$ - поперечная. Величина $\rho_{\mathrm{s} 12}$ отвечает за эффект увлечения сверхтекучим движением одной компоненты раствора другой компонентой, ранее обнаруженный в работах [9], [10]. Как видно из $(44)$, слагаемые в потоках числа частиц, пропорциональные $v_{\mathrm{s} 1 i}, v_{\mathrm{S} 2}$, приводят к эффекту увлечения каждой из компонент раствора. Кроме того, возникает новый физический механизм увлечения, связанный с влиянием магнитных степеней свободы на сверхтекучее движение. Слагаемые, пропорциональные вектору магнитной спирали q в плотностях потоков числа частиц, и слагаемые, пропорциональные $v_{\mathrm{s} a i}$ в плотности продольного потока спина, описывают этот эффект увлечения. В выражении для тензоpa натяжений слагаемые, пропорциональные $v_{\mathrm{s} 1 i} v_{\mathrm{s} 2 i}, v_{\mathrm{s} a i} v_{\mathrm{s} a i}$, отвечают за эффект увлечения одной свертекучей компоненты другой, а слагаемые, пропорциональные $v_{\mathrm{s} a i} q_{i}$, - за взаимное влияние орбитальных и спиновых степеней свободы. При получении формул (44) не предполагалось наличие инвариантности Галилея рассматриваемой физической системы.

В случае галилеевой инвариантности структура термодинамического потенциала как функции от термодинамических параметров $Y_{r}, p_{k}, q_{k}$ упрощается: $\omega\left(Y, \mathbf{p}_{a}, \mathbf{q}\right)=$ $\omega\left(Y^{\prime}, \mathbf{p}_{a}^{\prime}, \mathbf{q}^{\prime}\right)$. Здесь штрихованные параметры определяются равенствами

$$
\begin{gathered}
Y_{0}^{\prime}=Y_{0}, \quad Y_{\alpha}^{\prime}=Y_{\alpha}, \quad Y_{k}^{\prime}=Y_{k}+u_{i} Y_{0}, \quad Y_{a}^{\prime}=Y_{a}+m_{a} u_{i} Y_{i}+\frac{1}{2} m_{a} u^{2} Y_{0} \\
p_{a k}^{\prime}=p_{a k}-m_{a} u_{k} \quad(a=1,2), \quad q_{k}^{\prime}=q_{k},
\end{gathered}
$$

где $m_{a}$ - массы частищ, $u_{i}$ - параметр преобразования Галилея. В случае когда $u_{i}=$ $-Y_{i} / Y_{0} \equiv v_{\text {n } i}$, величина $Y_{k}^{\prime}$ обрашается в ноль. Если учесть в термодинамическом потенциале зависимость от скалярных инвариантов с точностью до квадратичных слагаемых, то будут справедливы соотношения, связываюшие введенные в (43) величины

$$
m_{1}^{*}=m_{1}, \quad m_{2}^{*}=m_{2}, \quad \rho=\rho_{\mathrm{n}}+\rho_{\mathrm{s} 1}+\rho_{\mathrm{s} 2}+2 \rho_{\mathrm{s} 12},
$$

где $m_{1}, m_{2}$ - массы частиц компонент раствора. 


\section{5. УРАВНЕНИЯ ГИДРОДИНАМИКИ С ВЕКТОРНЫМ ПП}

Рассмотрим вопрос о построении идеальной гидродинамики раствора двух сверхтекучих ферми-жидкостей, описываемых векторным ПП. В набор параметров сокращенного описания входят дополнительные термодинамические переменные, которые могут быть представлены в терминах ПП и произвольного неравновесного статистического оператора.

В векторном состоянии сверхтекучие фазы вводятся равенствами

$$
\varphi_{1,2}(\mathbf{x}, t)=\frac{1}{2} \operatorname{Im}\left\{\ln \left(\operatorname{Sp} \hat{f}(t) \hat{\Delta}_{\alpha}(\mathbf{x})\right)^{2} \pm \ln \left(\operatorname{Sp} \hat{f}(t) \hat{\Delta}_{\alpha}^{2}(\mathbf{x})\right)\right\}
$$

(индекс 1 соответствует верхнему знаку в формуле (45), а 2 - нижнему). Ортогональная матрица поворота $a(\mathbf{x}, t)$ может быть выражена через векторный ПП в неявном виде

$$
\mathbf{e}_{1} a(\mathbf{x}, \hat{f}(t)) \boldsymbol{\Delta}_{1}(\mathbf{x}, \hat{f}(t))=\mathbf{e}_{1} a(\mathbf{x}, \hat{f}(t)) \boldsymbol{\Delta}_{2}(\mathbf{x}, \hat{f}(t))=\mathbf{e}_{2} a(\mathbf{x}, \hat{f}(t)) \boldsymbol{\Delta}_{2}(\mathbf{x}, \hat{f}(t))=0
$$

Здесь $\mathbf{e}_{1}, \mathbf{e}_{2}$ - единичные взаимно ортогональные векторы, не зависящие от статистического оператора, и $\boldsymbol{\Delta}_{1}=\operatorname{Re} \boldsymbol{\Delta}, \boldsymbol{\Delta}_{2}=\operatorname{Im} \boldsymbol{\Delta}$.

Построим уравнения гидродинамики раствора двух сверхтекучих ферми-жидкостей. На гидродинамическом этапе эволюции этот раствор описывается сокрашенным набором параметров: плотностями аддитивных интегралов движения $\zeta$, сверхтекучими импульсами $\mathbf{p}_{a}$, ортогональной матрицей поворота $a$. Функциональная гипотеза в этом случае имеет вид

$$
\hat{f}(t) \underset{t \gg \tau_{0}}{\longrightarrow} \hat{f}\left(\zeta(t), \varphi_{a}(t), a(t)\right),
$$

где $\tau_{0}$ - время релаксации.

Запишем уравнения для величин $\zeta(t), \varphi_{a}(t), a(t)$ :

$$
\begin{aligned}
\dot{\zeta}_{r}\left(\mathbf{x}, \hat{f}\left(\zeta, \varphi_{a}, a\right)\right) & =i \operatorname{Sp} \hat{f}\left(\zeta, \varphi_{a}, a\right)\left[\hat{\varepsilon}\left(\hat{f}\left(\zeta, \varphi_{a}, a\right)\right), \hat{\zeta}_{r}\left(\mathbf{x}, \hat{f}\left(\zeta, \varphi_{a}, a\right)\right)\right] \\
\dot{a}_{\alpha \beta}\left(\mathbf{x}, \hat{f}\left(\zeta, \varphi_{a}, a\right)\right) & =i \operatorname{Sp} \hat{f}\left(\zeta, \varphi_{a}, a\right)\left[\hat{\varepsilon}\left(\hat{f}\left(\zeta, \varphi_{a}, a\right)\right), \hat{a}_{\alpha \beta}\left(\mathbf{x}, \hat{f}\left(\zeta, \varphi_{a}, a\right)\right)\right] \\
\dot{\varphi}_{a}\left(\mathbf{x}, \hat{f}\left(\zeta, \varphi_{a}, a\right)\right) & =i \operatorname{Sp} \hat{f}\left(\zeta, \varphi_{a}, a\right)\left[\hat{\varepsilon}\left(\hat{f}\left(\zeta, \varphi_{a}, a\right)\right), \hat{\varphi}_{a}\left(\mathbf{x}, \hat{f}\left(\zeta, \varphi_{a}, a\right)\right)\right]
\end{aligned}
$$

Входящие сюда операторы $\hat{a}_{\alpha \beta}, \hat{\varphi}_{a}$ определяются выражениями

$$
\delta a_{\alpha \beta}(\mathbf{x}, \hat{f})=\operatorname{Sp} \delta \hat{f} \hat{a}_{\alpha \beta}(\mathbf{x}, \hat{f}), \quad \delta \varphi_{a}(\mathbf{x}, \hat{f})=\operatorname{Sp} \delta \hat{f} \hat{\varphi}_{a}(\mathbf{x}, \hat{f}) .
$$

Рассмотрим уравнение для ортогональной матрищы поворота $a_{\alpha \beta}(48)$. Пренебрегая в нем релаксационными процессами, имеем

$$
\dot{a}_{\alpha \beta}\left(\mathbf{x}, \hat{f}_{0}\right)=i \operatorname{Sp} \hat{f}_{0}\left[\hat{\varepsilon}\left(\hat{f}_{0}\right), \hat{a}_{\alpha \beta}\left(\mathbf{x}, \hat{f}_{0}\right)\right]
$$

откуда, используя соотношения

$$
i \operatorname{Sp} \hat{f}\left[\hat{s}_{\alpha}\left(\mathbf{x}^{\prime}\right), \hat{a}_{\beta \gamma}(\mathbf{x}, \hat{f})\right]=\varepsilon_{\alpha \rho \gamma} a_{\beta \rho}(\mathbf{x}) \delta\left(\mathbf{x}-\mathbf{x}^{\prime}\right), \quad i \operatorname{Sp} \hat{f}\left[\hat{n}\left(\mathbf{x}^{\prime}\right), \hat{a}_{\alpha \beta}(\mathbf{x}, \hat{f})\right]=0,
$$


а также свойства симметрии (25), получим уравнение движения для ортогональной матрицы поворота $a_{\alpha \beta}$ в главном приближении

$$
\dot{a}_{\alpha \beta}=a_{\alpha \lambda} \varepsilon_{\lambda \beta \gamma}\left(h_{\gamma}+\mathbf{q} \mathbf{v}_{n} n_{\gamma}\right), \quad h_{\gamma} \equiv-\frac{Y_{\gamma}}{Y_{0}}, \quad v_{\mathrm{n} k} \equiv-\frac{Y_{k}}{Y_{0}} .
$$

Здесь $h_{\gamma}$ - внутреннее магнитное поле, $v_{\mathrm{n} k}$ - нормальная скорость.

Теперь выведем уравнение для сверхтекучих фаз $\varphi_{a}$. Пренебрегая в (49) релаксационными процессами, имеем

$$
\dot{\varphi}_{a}\left(\mathbf{x}, \hat{f}_{0}\right)=i \operatorname{Sp} \hat{f}_{0}\left[\hat{\varepsilon}\left(\hat{f}_{0}\right), \hat{\varphi}_{a}\left(\mathbf{x}, \hat{f}_{0}\right)\right] .
$$

Отсюда с учетом условий симметрии $(25)$ и свойств

$$
\begin{aligned}
& i \operatorname{Sp} \hat{f}\left[\hat{s}_{\alpha}\left(\mathbf{x}^{\prime}\right), \hat{\varphi}_{a}(\mathbf{x}, \hat{f})\right]=0, \quad i \operatorname{Sp} \hat{f}\left[\hat{n}\left(\mathbf{x}^{\prime}\right), \hat{\varphi}_{a}(\mathbf{x}, \hat{f})\right]=\delta\left(\mathbf{x}-\mathbf{x}^{\prime}\right), \\
& i \operatorname{Sp} \hat{f}\left[\hat{\pi}_{k}\left(\mathbf{x}^{\prime}\right), \hat{\varphi}_{a}(\mathbf{x}, \hat{f})\right]=\nabla_{k} \varphi_{a}(\mathbf{x}, \hat{f}) \delta\left(\mathbf{x}-\mathbf{x}^{\prime}\right)
\end{aligned}
$$

найдем уравнения движения для сверхтекучих фаз

$$
\dot{\varphi}_{a}=\mu_{a}+\mathbf{p}_{a} \mathbf{v}_{\mathrm{n}}, \quad \mu_{a} \equiv-\frac{Y_{a}}{Y_{0}},
$$

где $\mu_{a}$ - химические потенциалы.

В заключение выведем уравнения для аддитивных интегралов движения. Пренебрегая в (47) релаксационными процессами, получим

$$
\dot{\zeta}_{r}\left(\mathbf{x}, \hat{f}_{0}\right)=i \operatorname{Sp} \hat{f}_{0}\left[\hat{\varepsilon}\left(\hat{f}_{0}\right), \hat{\zeta}_{r}\left(\mathbf{x}, \hat{f}_{0}\right)\right]=-\nabla_{i} \operatorname{Sp} \hat{f}_{0} \hat{\zeta}_{r i}\left(\mathbf{x}, \hat{f}_{0}\right),
$$

где вид плотностей потоков аддитивных интегралов движения $\zeta_{r k}$ в терминах локально-равновесного термодинамического потенциала определяется формулами (32), (33), (36), (38).

Таким образом, мы получили полную систему уравнений гидродинамики раствора ферми-жидкостей в состоянии, описываемом векторным ПП. После перехода к новым переменным (40) эта система примет вид

$$
\begin{gathered}
\dot{\zeta}_{r}=-\nabla_{i} \underline{\zeta}_{r i}+\eta_{r}, \quad \dot{d}_{\alpha}=\varepsilon_{\alpha \beta \gamma}\left(\frac{\partial \varepsilon}{\partial \underline{s}_{\beta}}+\underline{z}_{\beta i} \frac{\partial \varepsilon}{\partial \pi_{i}}\right) d_{\gamma}, \\
\dot{p}_{a i}=-\nabla_{i}\left(\frac{\partial \varepsilon}{\partial n_{a}}+p_{a j} \frac{\partial \varepsilon}{\partial \pi_{j}}\right), \\
\dot{q}_{i}=-\nabla_{i}\left(d_{\alpha} \frac{\partial \varepsilon}{\partial \underline{s}_{\alpha}}+q_{j} \frac{\partial \varepsilon}{\partial \pi_{j}}\right)+\varepsilon_{\alpha \beta \gamma} d_{\alpha \underline{z}_{\beta i}}\left(\frac{\partial \varepsilon}{\partial \underline{s}_{\gamma}}+\underline{z}_{\gamma j} \frac{\partial \varepsilon}{\partial \pi_{j}}\right), \\
\underline{\dot{z}}_{\alpha i}=-\left(\delta_{\alpha \beta}-d_{\alpha} d_{\beta}\right) \nabla_{i}\left(\frac{\partial \varepsilon}{\partial \underline{s}_{\beta}}+\underline{z}_{\beta j} \frac{\partial \varepsilon}{\partial \pi_{j}}\right)+\varepsilon_{\alpha \beta \gamma} \underline{z}_{\beta i}\left(\frac{\partial \varepsilon}{\partial \underline{s}_{\gamma}}+\underline{z}_{\gamma j} \frac{\partial \varepsilon}{\partial \pi_{j}}\right), \\
\eta_{r}=\delta_{r \alpha} \varepsilon_{\alpha \beta \gamma}\left(\underline{\zeta}_{\gamma k}\left(q_{k} d_{\alpha}+\underline{z}_{\beta k}\right)+\underline{\zeta}_{\gamma}\left(q_{0} d_{\alpha}+\underline{z}_{\beta 0}\right)\right),
\end{gathered}
$$


где $\eta_{r}$ представляет собой источник в уравнении для спина. Заметим, что в отличие от плотности спина $s_{\alpha}$ плотность $\underline{s}_{\alpha}=a_{\alpha} s_{\beta}$ не является плотностью адлитивного интеграла движения, и поэтому в уравнениях гидродинамики (50) возникает источник. Удобство записи уравнений (50) и основного соотношения термодинамики (35) заключается в том, что они сформулированы в терминах термодинамических переменных, не зависящих от координат и времени в состоянии равновесия. В этом случае возможно построение простой теории возмушений по малым пространственным неоднородностям для всех параметров сокрашенного описания.

Уравнения идеальной гидродинамики (50), второе начало термодинамики (35) и плотности потоков аддитивных интегралов движения (42) описывают неравновесную динамику раствора сверхтекучих ферми-жидкостей с векторным ПП.

Уравнения гидродинамики и основные термодинамические соотношения для раствора сверхтекучих ферми-жидкостей в состоянии с векторным ПП отличаются от известных выражений для сверхтекучей ферми-жидкости с синглетным спариванием [1], [2], [13] наличием в них переменных, связанных со второй компонентой раствора и с нарушением симметрии относительно спиновых врашений (вектора магнитной спирали $q_{i}$, поперечной формы Картана $z_{\alpha i}$ и вектора магнитной анизотропии $\left.d_{\alpha}\right)$. Если в полученных уравнениях гидродинамики и термодинамических соотношениях исключить плотность числа частиц второй компоненты и ее сверхтекучий импульс, а также заменить вектор магнитной спирали $q_{i}$, поперечную форму Картана $z_{\alpha i}$, вектор магнитной анизотропии $d_{\alpha}$ на форму Картана $\underline{\omega}_{\alpha i}$, то эти уравнения совпадут с аналогичными уравнениями для сверхтекучей ферми-жидкости в состоянии с триплетным ПП [5], [14]. Рассматриваемый в этой работе раствор описывается набором параметров, включающим в себя, кроме переменных раствора ферми-жидкостей с синглетным спариванием [9], [11], также переменные, связанные со спонтанным нарушением симметрии при спиновых врашениях.

Рассмотрим вопрос о коллективных возбуждениях в описываемом растворе фермижидкостей. Линеаризуя систему уравнений (50) вблизи состояния равновесия, получим дисперсионное уравнение

$$
\sum_{m=0}^{14} A_{m}(k) \omega^{m}=0,
$$

где коэффициенты $A_{m}(k)$ представляют собой комбинации из производных от $\varepsilon$ по всем переменным.

Так как дисперсионное уравнение является достаточно сложным, то для выявления особенностей спектров рассмотрим модельный функционал энергии

$$
\begin{aligned}
\varepsilon= & \varepsilon_{0}\left(n_{1}, n_{2}, \sigma\right)+\frac{1}{2 \rho} \pi^{2}+\frac{1}{2} \alpha_{1} p_{1}^{2}+\frac{1}{2} \alpha_{2} p_{2}^{2}+\alpha_{3} \mathbf{p}_{1} \mathbf{p}_{2}+ \\
& +\frac{1}{2 \chi} \underline{s}^{2}+\frac{1}{2} \tau \underline{\omega}^{2}+\kappa \mathbf{p}_{1} \underline{\omega}_{\alpha} \mathbf{p}_{2} \underline{\omega}_{\alpha}
\end{aligned}
$$

содержащий квадратичные слагаемые по параметрам сокращенного описания и только одно слагаемое четвертого порядка, отвечающее за взаимодействие между подсистемами. 
Рассмотрим три частных случая.

1. $\mathbf{p}_{1}=0, \mathbf{p}_{2}=0, \underline{\omega}=0, \mathbf{s}=0$. В результате несложных вычислений получим дисперсионное уравнение

$$
\omega^{2}\left(\omega^{6}-A_{1} k^{2} \omega^{4}+A_{2} k^{4} \omega^{2}-A_{3} k^{6}\right)\left(\omega^{2}-B k^{2}\right)^{3}=0, \quad k=|\mathbf{k}|,
$$

где

$$
\begin{gathered}
A_{1}=a_{1}+b_{2}+c_{3}, \quad A_{2}=a_{1} b_{2}+a_{1} c_{3}+b_{2} c_{3}+a_{3} c_{1}+a_{2} b_{1}+c_{2} b_{3}, \\
A_{3}=a_{1} b_{2} c_{3}+a_{2} b_{3} c_{1}+a_{3} b_{1} c_{2}-a_{3} b_{2} c_{1}-a_{2} b_{1} c_{3}-a_{1} b_{3} c_{2}, \\
B=\frac{\tau}{\chi}, \quad a_{1}=\frac{v \sigma^{0}}{\rho}, \quad a_{2}=\frac{u_{1} \sigma^{0}}{\rho}, \quad a_{3}=\frac{u_{2} \sigma^{0}}{\rho}, \quad b_{1}=\frac{v n_{1}^{0}}{\rho}+\alpha_{1} \xi_{1}+\alpha_{3} \xi_{2}, \\
b_{2}=\frac{u_{1} n_{1}^{0}}{\rho}+\alpha_{1} \zeta_{1}+\alpha_{3} \lambda, \quad b_{3}=\frac{u_{2} n_{1}^{0}}{\rho}+\alpha_{1} \lambda+\alpha_{3} \zeta_{2}, \quad u_{1}=\frac{\partial p}{\partial n_{1}}, \\
c_{1}=\frac{v n_{2}^{0}}{\rho}+\alpha_{3} \xi_{1}+\alpha_{2} \xi_{2}, \quad c_{2}=\frac{u_{1} n_{2}^{0}}{\rho}+\alpha_{3} \zeta_{1}+\alpha_{2} \lambda, \quad u_{2}=\frac{\partial p}{\partial n_{2}}, \\
c_{3}=\frac{u_{2} n_{2}^{0}}{\rho}+\alpha_{3} \lambda+\alpha_{2} \zeta_{2}, \quad v=\frac{\partial p}{\partial \sigma}, \quad \xi_{1}=\frac{\partial^{2} \varepsilon}{\partial n_{1} \partial \sigma}, \quad \xi_{2}=\frac{\partial^{2} \varepsilon}{\partial n_{2} \partial \sigma}, \\
\zeta_{1}=\frac{\partial^{2} \varepsilon}{\partial n_{1}^{2}}, \quad \zeta_{2}=\frac{\partial^{2} \varepsilon}{\partial n_{2}^{2}}, \quad \lambda=\frac{\partial^{2} \varepsilon}{\partial n_{1} \partial n_{2}} .
\end{gathered}
$$

Из уравнения (52) найдем нетривиальные решения

$$
\begin{gathered}
\omega_{1}^{2}=w_{1}^{2} k^{2}, \quad \omega_{2}^{2}=w_{2}^{2} k^{2}, \quad \omega_{3}^{2}=w_{3}^{2} k^{2}, \\
w_{1}^{2}+w_{2}^{2}+w_{3}^{2}=A_{1}, \quad w_{1}^{2} w_{2}^{2}+w_{1}^{2} w_{3}^{2}+w_{2}^{2} w_{3}^{2}=A_{2}, \\
w_{1}^{2} w_{2}^{2} w_{3}^{2}=A_{3}, \quad \omega_{4,5,6}^{2}=B k^{2} .
\end{gathered}
$$

Они представляют собой три ветви звуковых колебаний и вырожденные спиновые моды. В приближении $w_{1}^{2} \gg w_{2}^{2} \gg w_{3}^{2}$, используемом в работах [9] для получения спектров звуковых колебаний, скорости звуковых мод примут вид

$$
w_{1}^{2}=A_{1}, \quad w_{2}^{2}=\frac{A_{2}}{A_{1}}, \quad w_{3}^{2}=\frac{A_{3}}{A_{2}} .
$$

Колебания $\omega_{1}^{2}, \omega_{2}^{2}, \omega_{3}^{2}$ соответствуют первому, второму и третьему звукам. В случае малых сверхтекучих скоростей и концентрации раствора формулы (54) дадут результаты работ [9] по гидродинамике растворов сверхтекучих жидкостей с синглетным спариванием.

2. $\mathbf{p}_{1}=\mathbf{p}_{2}=0, \underline{\omega}_{\alpha i}=n_{\alpha} q_{i} \neq 0, \mathbf{s}=0$, вектор $\mathbf{q}$ параллелен $\mathbf{k}$ и мал. В этом случае звуковые моды можно записать в виде

$$
\begin{gathered}
\omega_{1}^{2}=w_{1}^{2} k^{2}, \quad \omega_{2}^{2}=w_{2}^{2} k^{2}, \quad \omega_{3}^{2}=w_{3}^{2} k^{2}, \\
w_{1}^{2}+w_{2}^{2}+w_{3}^{2}=A_{1}^{\prime}, \quad w_{1}^{2} w_{2}^{2}+w_{1}^{2} w_{3}^{2}+w_{2}^{2} w_{3}^{2}=A_{2}^{\prime}, \\
w_{1}^{2} w_{2}^{2} w_{3}^{2}=A_{3}, \quad A_{1}^{\prime}=A_{1}+\kappa\left(\zeta_{1}+\lambda\right) q^{2}, \\
A_{2}^{\prime}=A_{2}+A_{1} \kappa\left(\zeta_{1}+\lambda\right) q^{2}-\left(A_{3} \xi_{1}-A_{2} \xi_{1}\right) q^{2} .
\end{gathered}
$$


В приближении $w_{1}^{2} \gg w_{2}^{2} \gg w_{3}^{2}$ скорости звуковых мод:

$$
w_{1}^{2}=A_{1}^{\prime}, \quad w_{2}^{2}=\frac{A_{2}^{\prime}}{A_{1}^{\prime}}, \quad w_{3}^{2}=\frac{A_{3}}{A_{2}^{\prime}}
$$

спиновые колебания:

$$
\omega_{1}^{2}=B k^{2}, \quad \omega_{2,3}^{2}=B_{ \pm} k^{2} \pm B_{2}, \quad B_{ \pm}=B \pm B_{2}, \quad B_{2}=\frac{\tau}{\chi} q
$$

Как видно из формул (55), (56), в рассмотренной модели отличие от нуля вектора магнитной спирали q приводит к перенормировке скоростей звуков и снятию вырождения спиновых мод.

3. $\mathbf{p}_{1} \neq 0, \mathbf{p}_{2} \neq 0, \underline{\omega}=0, \mathbf{s}=0$, векторы $\mathbf{p}_{1,2}$ параллельны $\mathbf{k}$ и малы. Звуковые моды имеют вид

$$
\begin{aligned}
& \omega_{j}=w_{j} k, \quad j, k, l, m, n=1,2,3,4,5,6, \quad \sum_{j} w_{j}=0, \quad \sum_{j \neq k} w_{j} w_{k}=-A_{1}^{\prime \prime}, \\
& \sum_{j \neq k \neq l} w_{j} w_{k} w_{l}=A_{2}^{\prime \prime}, \quad \sum_{j \neq k \neq l \neq m} w_{j} w_{k} w_{l} w_{m}=-A_{3}^{\prime \prime}, \\
& \sum_{j \neq k \neq l \neq m \neq n} w_{j} w_{k} w_{l} w_{m} w_{n}=A_{4}^{\prime \prime}, \quad w_{1} w_{2} w_{3} w_{4} w_{5} w_{6}=-A_{5}^{\prime \prime}, \\
& A_{1}^{\prime \prime}=A_{1}+F_{3}^{\prime} H_{1}^{\prime}+F_{2}^{\prime} G_{1}^{\prime}, \quad A_{2}^{\prime \prime}=F_{3}^{\prime} H_{1}+F_{2}^{\prime} G_{1}+F_{3} H_{1}^{\prime}+F_{2} G_{1}^{\prime}, \\
& A_{3}^{\prime \prime}=A_{2}-G_{3} F_{2}^{\prime} H_{1}^{\prime}+G_{2} F_{3}^{\prime} H_{1}^{\prime}-H_{2} F_{3}^{\prime} G_{1}^{\prime}+H_{3} F_{2}^{\prime} G_{1}^{\prime} \text {, } \\
& A_{4}^{\prime \prime}=G_{3} F_{2} H_{1}^{\prime}+G_{3} F_{2}^{\prime} H_{1}-G_{2} F_{3} H_{1}^{\prime}-G_{2} F_{3}^{\prime} H_{1}+H_{2} F_{3} G_{1}^{\prime}+ \\
& +H_{2} F_{3}^{\prime} G_{1}-H_{3} F_{2} G_{1}^{\prime}-H_{3} F_{2}^{\prime} G_{1}, \quad A_{5}^{\prime \prime}=A_{3}, \\
& F_{2}^{\prime}=\alpha_{1} p_{1}+\alpha_{3} p_{2}, \quad F_{3}^{\prime}=\alpha_{3} p_{1}+\alpha_{2} p_{2}, \quad G_{1}^{\prime}=\frac{p_{1}}{\rho}, \quad H_{1}^{\prime}=\frac{p_{2}}{\rho}, \\
& F_{2}=\alpha_{1} u_{1}+\alpha_{3} u_{2}, \quad F_{3}=\alpha_{3} u_{1}+\alpha_{2} u_{2}, \\
& G_{1}=\frac{\sigma^{0}}{\rho} \xi_{1}+\frac{n_{1}^{0}}{\rho} \zeta_{1}+\frac{n_{2}^{0}}{\rho} \lambda, \quad G_{2}=\alpha_{1} \zeta_{1}+\alpha_{3} \lambda, \quad G_{3}=\alpha_{3} \zeta_{1}+\alpha_{2} \lambda, \\
& H_{1}=\frac{\sigma^{0}}{\rho} \xi_{2}+\frac{n_{1}^{0}}{\rho} \lambda+\frac{n_{2}^{0}}{\rho} \zeta_{2}, \quad H_{2}=\alpha_{1} \lambda+\alpha_{3} \zeta_{2}, \quad H_{3}=\alpha_{3} \lambda+\alpha_{2} \zeta_{2} .
\end{aligned}
$$

Спиновые моды: $\omega^{2}=\left(B+B_{1}\right) k^{2}, \quad B_{1}=(2 \kappa / \chi) p_{1} p_{2}$. В этом случае звуковые моды будут модифицированы по сравнению со случаем 1 , а скорости спиновых волн будут перенормированы в силу указанного вьше эффекта увлечения $\left(p_{a k} \neq 0\right)$.

На основании вышеизложенного можно заключить, что рассмотренная в этой работе система частично обладает как чертами раствора сверхтекучих жидкостей с синглетным спариванием, так и магнитной составляющей сверхтекучей ферми-жидкости с триплетным спариванием. 


\section{Список литературы}

[1] И. М. Халатников. Теория сверхтекучести. М.: Наука, 1971.

[2] Н. Н. Боголюбов. Препр. ОИЯИ Р-1395. Дубна, 1962.

[3] G. E. Volovik. Exotic Properties of Superfluid ${ }^{3}$ He. Singapore: World Scientific, 1992.

[4] D. Vollhardt, P. Wölfle. The Superfluid Phases of Helium 3. London: Ed. Taylor \& Francis, 1990.

[5] A. J. Leggett. Rev. Mod. Phys. 1975. V. 47. P. 331.

[6] R. Combescot. J. Phys. C. 1981. V. 14. P. 1619.

[7] K. Maki, P. Kumar. Phys. Rev. B. 1977. V. 16. P. 182.

[8] Сверхтекучесть гелия-3. Сб. статей. Ред. И.М. Халатников. М.: Мир, 1977.

[9] А.Ф. Андреев, Е. П. Башкин. ЖЭТФ. 1975. Т. 69. С. 319.

[10] М. Ю. Ковалевский, Н. М. Лавриненко. ФНТ. 1982. Т. 8. С. 341.

[11] Н. Н. Боголюбов, М. Ю. Ковалевский, А. М. Курбатов, С. В. Пелетминский, А. Н. Тарасов. УФН. 1989. Т. 159. С. 585.

[12] Л. Д. Ландау. Сб. трудов. Т. 1. М.: Наука, 1969. С. 128; 252.

[13] В. В. Красильников, С. В. Пелетминский, А.А. Рожков, А. А. Яценко. ЭЧАЯ. 1988. T. 19. C. 1440.

[14] М.Ю. Ковалевский, А. А. Рожков. ТМФ. 1997. Т. 113. С. 313.

[15] В. В. Красильников, А.А. Рожков, А. А. Яченко. ФНТ. 1990. Т. 16. С. 1368.

[16] M. Yu. Kovalevskii, A. A. Rozhkov. Physica A. 1995. V. 216. P. 169.

[17] Ю. М. Полуәктов, В. В. Красильников. ФНТ. 1989. Т. 15. С. 1251

[18] А.И. Ахиезер, С. В. Пелетминский, А.А. Яценко. ФНТ. 1994. Т. 20. С. 650.

[19] M. Baldo, U. Lombardo, P. Schuck. Phys. Rev. C. 1995. V. 52. P. 975.

[20] L. Amundsen, E. Ostgaard. Nucl. Phys. A. 1985. V. 442. P. 163.

[21] Th. Alm, G. Roepke, A. Sedrakian. Nucl. Phys. A. 1995. V. 594. P. 355.

[22] В. П. Минеев. УФН. 1983. Т. 139. С. 303.

[23] Э. Картан. Геометрия групп Ли и симметрические пространства. М.: ИЛ, 1949.

[24] Н. Н. Боголюбов. Квазисредние в задачах статистической механики. Преп. ОИЯИ Д-781. Дубна, 1961. 